\title{
Recovery of Link Quality Degradation in Wireless Mesh Networks
}

\author{
V. Khanna ${ }^{1 *}$, Krishna Mohanta ${ }^{2}$ and T. Saravanan ${ }^{3}$ \\ 1Department of CSE, Sri Sai Ram Engg. College, Leo Nagar, Chennai-600 044, India; drvkannan62@yahoo.com \\ 2Department of ECE, Bharath University Chennai-600073, India; krishnamohanta@gmail.com \\ 3Professor \& Head, Bharath University, Chennai, pci_saravanan@yahoo.cp.in
}

\begin{abstract}
The multiple system loops refers to a two pair of particular radios on both side of the loop. But, multiple system loops wireless mesh networks occurred often link failures because of channel clashing, obstacles, and/or based on demand of bandwidth requirement. This cause major efficiency degradation in WMNs or more expensive manual network management. This paper deals about Autonomous Link Recovery System. This makes a Multi-radio WMN automatically recall its local network channel and radio. Autonomous Link Recovery System has been implemented on ns2 based simulation and improvement of $90 \%$ efficiency.
\end{abstract}

Keywords: QoS-aware Planning Autonomous Link Recovery System (ALR).

\section{Introduction}

The applications of WMN are environmental management public interest, and worldwide wireless Internet services [1-3]. Wireless mesh network is made built up using radio nodes compiled in a mesh topology. Clients mesh routers, Laptops, cell phones and gateways are the main parts of Wireless mesh networks. They have in various forms (e.g., using multiradio/channel systems [4-7]) to meet the increasing capacity demands. Because of heterogeneous and fluctuating wireless link [8-10], pre assume the required performance of such WMNs is still a problem.

They are several solutions have been proposed First, resource-allocation algorithms [12-14] can provide (theoretical) guidelines for initial network resource planning. Second one is a channel-assignment using greedy algorithm. Changing settings of the faulty link(s) this problem can be reducible. Third, fault-tolerant routing protocols, such as local rerouting [16] or multipath routing [17].
This problem can be adjusted by using network-level path diversity.

Figure [1] Explain about the initial assignment of frequency channels as shown. The network often experiences wireless link failure and needs to reconfigure its settings.

These limitations can be over come by; this paper proposes an autonomous link recovery system (ALR). Next, based on the generated configuration changes, the system automatically reassigns the N/W settings among local mesh routers. Then, by imposing current network settings as constraints, ALR conclude the reassigned plans. Then it changes the network with minimum requirement for good network system. Briefly, ALR first searches for feasible local configuration changes available around a faulty area, based on current channel and radio associations.

The first proposal also includes a monitoring protocol using that conjunction with the planning algorithm. The accurate link-quality information is used to identify changes of network that satisfy applications' new QoS demands.

*Corresponding author:

V. Khanna (drvkannan62@yahoo.com) 
First, ALR's planning algorithm effectively concludes the reconfiguration network, QoS demands, accommodating twice more flows than static assignment. Next, ALR avoids the ripple effect via QoS-aware reconfiguration planning assignment. Section 2 deals about the design rationale and algorithms of ALR. Section 3 gives the in-depth simulation results of ALR. Section 4 gives the conclusion of paper.

ALR first generates feasible changes of each link, and then combines a set of feasible changes. Starting from a faulty link(s), ALR considers link changes within the first hops and generates feasible plans. Figure 1 explains about network often experiences wireless link failure and needs to reconfigure its settings. The total number of reconfiguration changes is determined on the basis of existing configurations around the faulty. If ALR cannot find a local solution, it increases the number of hops so that ALR may explore a broad range.

\section{Architecture}

The ALR architecture has been explained detailed as follows

\subsection{Overview}

ALR is a distributed control system. This is easily deployable in IEEE 802.11-based mr-WMNs.

This generates reconfiguration plans Based on multiple channels and radio configuration available. This allow for changes of network configurations only in where link failures occurred.

ALR effectively identifies QoS-certifiable reconfiguration plans by: 1) estimating the QoS-stability of generated reconfiguration plans. 2) utilization of expected benefits in channel.

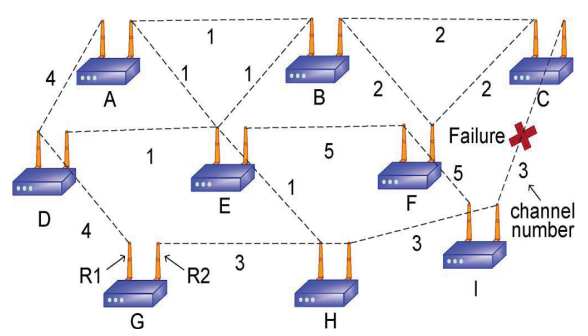

Figure 1. Multiradio WMN.AWMNhas an initial assignment of frequency channels as shown. The network often experiences wireless link failure and needs to reconfigure its settings.
Algorithm 1: ALR Operation at mesh node: $i$

(1) Monitoring period $\left(t_{m}\right)$

1 : for every link $j$ do

2: measure link-quality $(l q)$ using passive monitoring;

3: end for

4: send monitoring results to a gateway $g$;

(2) Failure detection and group formation period $\left(t_{f}\right)$

5: if link $l$ violates link requirements $r$ then

6: request a group formation on channel $c$ of link $l$;

7: end if

8: participate in a leader election if a request is received;

(3) Planning period $\left(M, t_{p}\right)$

9: if node $i$ is elected as a leader then

10: send a planning request message $(c, M)$ to a gateway;

11: else if node $i$ is a gateway then

12: synchronize requests from reconfiguration groups $M_{\mathrm{n}}$

13: generate a reconfiguration plan $(p)$ for $M_{i}$;

14: send a reconfiguration plan $(p)$ to a leader of $M_{i}$;

15: end if

(4) Reconfiguration period $\left(p, t_{r}\right)$

16: if $p$ includes changes of node $i$ then

17: apply the changes to links at $t$;

18: end if

19: relay $p$ to neighboring members, if any

ALR exactly monitors the quality of each node in a distributed manner. Furthermore, based on the measurements and given links' QoS constraints, ALR autonomously initiates network reconfiguration when local link failures occur. Figure 2 explain about Localized reconfiguration planning process in ALR.

This network actively interacts with the other network and link layer for planning. This enables ALR to include a rerouting for reconfiguration planning in addition to linklayer reconfiguration. ALR can also maintain connectivity during recovery.

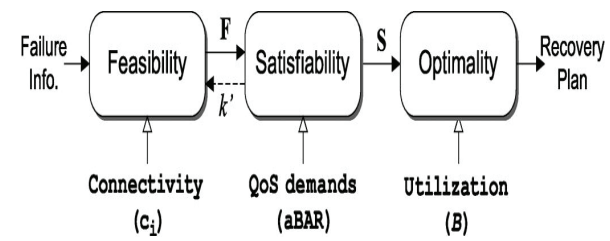

Figure 2. Localized reconfiguration planning process in ALR. 
We assume that during the formation and reconfiguration, all messages are reliably delivered via a routing protocol and per-hop retransmission timer

\subsection{Planning for Localized Network}

As a set of links' configuration changes, necessary for a network to recover from a link(s) failure on a channel is called as reconfiguration plan [11]. There are usually multiple reconfiguration plans for each link failure. Existing channel-assignment and scheduling algorithms $[5,12,13]$ seek "optimal" solutions by considering tight QoS constraints on all links. This require a huge configuration space to be searched and hence making the planning often an NP-complete problem [5]. ALR applies strict constraints to detect a reconfiguration plan that satisfies the QoS demands and it should be improves network utilization by contrast. ALR systematically generates reconfiguration plans that localize network changes by dividing the reconfiguration planning into three processes-feasibility, QoS satisfiability, and optimality.

ALR first has to ensure that the faulty link, which is needs to be fixed via reconfiguration. To this end, ALR considers three primitive link changes to fix a faulty link(s), ALR can use: 1) simultaneously change their tuned channel; 2) one radio in node A can switch its channel and associate with another radio in node B 3) use a detour path instead of the faulty link.

Generating feasible plans is necessary to find all legitimate changes in links' configurations and their combinations in faulty area. Given multiple radios, channels, and routes, in generating such plans, ALR has to face the following challenges.

While avoiding the use of the faulty channel, ALR needs to maintain connectivity with the full utilization of radio resources. Because each radio can associate itself with multiple neighboring nodes, a change in one link triggers other neighboring links to change their settings.

Let us consider an illustrative example in Figure 3. Given the failure in link CI, ALR first generates feasible and desirable changes per link (gray columns) using the primitives. The changes must not include the use of a faulty redundant channel. Then, ALR combines the generated per-link primitives of neighboring links for feasible plans. [15]

During the combination, ALR has to preserve link and/ or radio connectivities. For example, plans $S(C, I)_{3 \rightarrow 6}$ and $S(H, I)_{3 \rightarrow 3}$ in Figure 3 cannot be connected because each

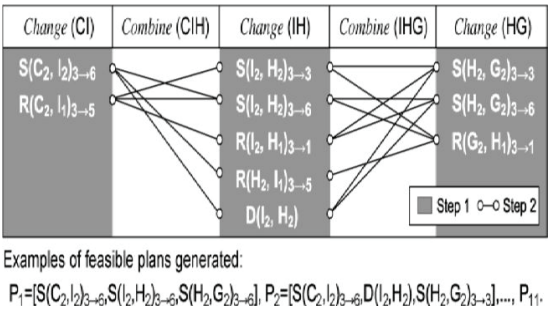

Figure 3. Example of network planning. ALR generates per-link changes (gray columns) and then connects them for feasible reconfiguration plans (white columns) for recovery of the failure in Figure 1.

change requires the same radio of node $I$ to set up different channels. After the two steps, ALR has 11 feasible reconfiguration plans $(F)$ by traversing connected changes of all links considered in the planning. Note that we set $k$ to 2 in this example.

ALR has to limit network changes as local as possible, but at the same time it needs to find a locally optimal solution by considering more network changes or scope. To make this tradeoff, ALR uses a $k$-hop reconfiguration parameter. Starting from a faulty link(s), ALR considers link changes within the first $\mathrm{k}$ hops and generates feasible plans. If ALR cannot find a local solution, it increases the number of hops (k) so that ALR may explore a broad range of link changes. Thus, the total number of reconfiguration changes is determined on the basis of existing configurations around the faulty area as well as the value of $k$.

Among a set of feasible plans F, ALR now needs to identify QoS-satisfying reconfiguration plans by checking if the QoS constraints are met under each plan. Although each feasible plan ensures that a faulty link(s) will use nonfaulty channels and maintain its connectivity, some plans might not satisfy the QoS constraints or may even cause cascaded QoS failures on neighboring links. To filter out such plans, ALR has to solve the following challenges.

Even though numerous bandwidth-estimation techniques have been proposed, they focus on the average bandwidth of each node in a network $[23,30]$ or the endto-end throughput of flows [17], which cannot be used to calculate the impact of per-link configuration changes. By contrast, ALR estimates an individual link's capacity $C$ based on measured (or cached) link-quality information packet-delivery ratio and data-transmission rate measured 
by passively monitoring the transmissions of data or probing packets [31]. Here, we assume that ALR is assumed to cache link-quality information for other channels and use the cached information to generate reconfiguration plans. If the information becomes obsolete, ALR detects link failures and triggers another reconfiguration to find QoScertifiable plans via lazy monitoring.

Given measured bandwidth and bandwidth requirements, ALR has to check if the new link change(s) satisfies QoS requirements. ALR defines and uses the expected busy airtime ratio of each link to check the link's QoS satisfiability. Assuming that a link's bandwidth requirement $q$ is given, the link's busy airtime ratio (BAR) can be defined as $B A R=q / C$ and must not exceed 1.0 (i.e., $B A R<1.0$ ) for a link to satisfy its bandwidth requirement. If multiple links share the airtime of one channel, ALR calculates aggregate BAR $a B A R$ of end-radios of a link, which is defined as $\operatorname{aBAR}(k)=\sum_{l \in L(k)}\left(q_{l} / C_{l}\right)$, where $\mathrm{k}$ is a radio ID, a link associated with radio $k$, and $L(k)$ the set of directed links within and across radio $k$ 's transmission range.

Besides the link change, ALR needs to check whether neighboring links are affected by local changes (i.e., cascaded link failures). To identify such adverse effect from a plan, ALR also estimates the QoS-satisfiability of links one hop away from member nodes whose links' capacity can be affected by the plan. If these one-hop-away links still meet the QoS requirement, the effects of the changes do not propagate thanks to spatial reuse of channels. Otherwise, the effects of local changes will propagate, causing cascaded QoS failures.

Let us consider an example in Figure 4. Assuming BAR of each directed link $\left(l_{i}\right)$ is 0.2 (e.g., $2 \mathrm{Mb} / \mathrm{s} 10 \mathrm{Mb} / \mathrm{s}$ ) in a tuned channel, a BAR of each radio tuned to channel 1 does not exceed 1.0, satisfying each link's QoS requirement. In

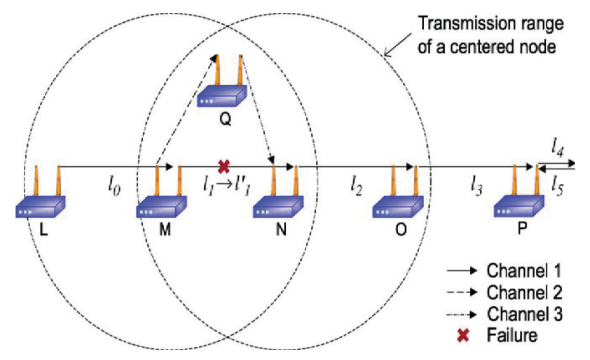

Figure 4. Busy airtime ratio (BAR) of a directed link. BAR (e.g., $\left.l_{1}\right)$ is affected by activities of neighboring links $\left(l_{0}, l_{2}\right.$, and $l_{3}$ ) in channel 1 and is used to evaluate QoS satisfiability of a link. addition, assuming that $B A R\left(l_{1}\right)$ increases from 0.2 to 0.4 in Figure 4. To accommodate this increase, reconfiguration plans that have a detour path through node $\mathrm{Q}$ do not affect the QoS-satisfiability of the neighboring nodes. On the other hand, plans with radio switches (e.g. $\left.\mathrm{R}\left(\mathrm{L}_{2}, \mathrm{M}_{1}\right)_{1 \rightarrow 2}\right)$ satisfy the QoS of link MN but cause a $B A R\left(l_{1}\right)$ to exceed 1.0, resulting in cascaded QoS failures of links beyond node $\mathrm{O}$.

ALR now has a set of reconfiguration plans that are QoS-certifiable and needs to choose a plan within the set for a local network to have evenly distributed link capacity. However, to incorporate the notion of fair share into the planning, ALR needs to address the following challenges.

ALR has to quantify the potential changes in link-capacity distribution from a plan. To this end, ALR defines and uses a benefit function $B(p)$ that quantifies the improvement of channel utilization that the reconfiguration plan $p$ makes. Specifically, the benefit function is defined as $B(p)=$ $(1 / n) \sum_{k=1}^{n}(k) \sum_{k=1}^{n}(k)$, where $\beta(k)$ is the relative improvement in the airtime usage of radio $k$, and $n$ the number of radios whose $\beta(k)$ has changed from the plan. This definition allows the benefit function to quantify the overall change in airtime usage, resulting from the reconfiguration plan. Here, $\beta(k)$ is considered as a fairness index on the usage of channel airtime, and it is defined as follows:

where $\epsilon_{1}(k)$ and $\epsilon_{2}(k)$ are estimated a $B A R$ 's of a radio $\mathrm{k}$ in existing configurations and in new configurations, respectively, and the desired channel utilization.

Multiple reconfiguration plans can have the same benefit, and ALR needs to break a tie among them. ALR uses the number of link changes that each plan requires to break a tie. Although link configuration changes incur a small amount of flow disruption (e.g., in the order of $10 \mathrm{~ms}$ ), the less changes in link configuration, the less network disruption.

Suppose $\delta$ is 0.5 as shown in Figure 5. ALR favors a plan that reconfigures links to have $50 \%$ available channel

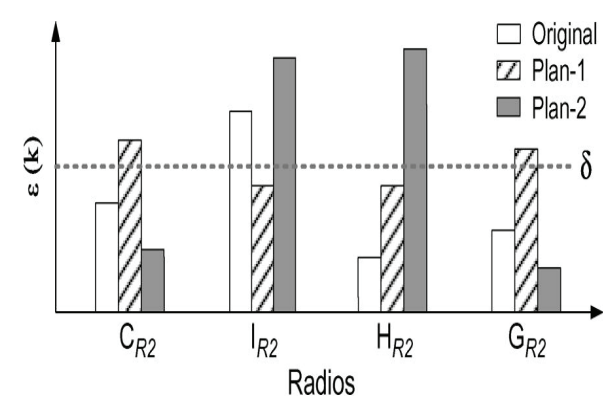

Figure 5. Benefit function. Prefers a reconfiguration plan that improves overall channel utilization close to the desired parameter. 
airtime (e.g., plan 1 in the figure). If a plan reconfigures a WMN to make the links heavily utilized while idling others (e.g., plan 2), then the benefit function considers the plan ineffective, placing the plan in a lowly ranked position. The effectiveness of $\beta$ and $\delta$ will be evaluated and discussed further in Section V-B2 implies that if a reconfiguration plan makes overall links' channel utilization closer to the desired utilization $\delta$, then $\beta(k)$ gives a positive value, while giving a negative value otherwise.

\subsection{Complexity of ALR}

Because of distributed and localized design, ALR have reasonable bandwidth and computation overheads. First, the network monitoring part in the reconfiguration protocols is made highly efficient and consumes less than $12 \mathrm{~kb} / \mathrm{s}$ probing bandwidth.

Next, the computational overhead in ALR mainly stems from the planning algorithms. Specifically, generating its possible link plans incurs $O(n+m)$ complexity, where $n$ is the number of available channels and $m$ the number of radios.

Next, a gateway node needs to generate and evaluate feasible plans, which have to search overhead in a constraint graph that consists of $O(l(n+m))$ nodes, where $l$ is the number of links

\section{Evaluation of ALR}

We first discuss about the simulation methodology, and then present the evaluation results on ALR.

ns-2 [37] is used in our simulation study. Throughout the simulation, we use a grid topology with 25 nodes in an area of $1 \times 1 \mathrm{~km} 2$, as shown in Figure 6(a). In the topology, adjacent nodes are separated by $180 \mathrm{~m}$, and each node is

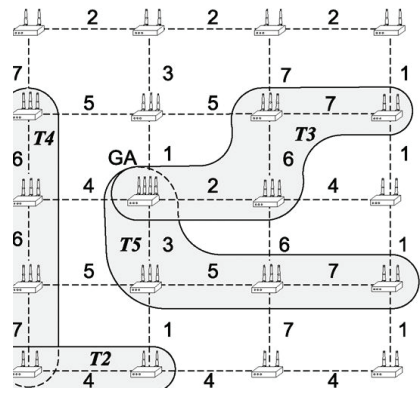

Figure 6. Satisfying varying QoS constraints. (a) Requests with different QoS requirements. (b) Improved (or changed) network capability (i) before and (ii) after reconfiguration. equipped with a different number of radios, depending on its proximity to a gateway. The gateway is equipped with four radios, one-hopaway nodes from a gateway have three radios, and other nodes have two radios.

We measured the effectiveness of ALR in meeting the varying QoS requirements in a mr-WMN. We initially assign symmetric link capacity as shown in the channel assignment of the grid topology [Figure 6(a)]. Then, while changing the QoS constraints in gray areas at different times (i.e.T1,...,T5 ), we evaluate the improvement of available capacity that ALR can generate via reconfiguration. As shown in the tables of Figure 6(b), ALR reconfigures a wireless mesh network to meet different QoS requirements. Before each reconfiguration, the gray areas can only accept 1-9 UDP flows. On the other hand, after reconfiguration, the network in the areas can admit 4-15 additional flows, improving the average network capacity of the gray areas by 3.5 times.

We also studied the impact of the benefit function on the ALR's planning algorithm. We conducted the same experiment as the previous one with different values of $\delta$ in the benefit function. As shown in Figure 6(b), a high value (0.8) of $\delta$ allows ALR to keep local channel efficiency high. By contrast, a low value (0.4) can deliver more available bandwidth (on average, $1.2 \mathrm{Mb} / \mathrm{s}$ ) than when the high value is used since ALR tries to reserve more capacity.

We evaluated the impact of the reconfiguration range. We used the same experiment settings as the previous one and focused on reconfiguration requests at T1. As we increase the hop count $\mathrm{k}$ from a faulty link(s), we measure the capacity improvement achieved by the reconfiguration plans. In addition, we calculate the capacity gain per change as the cost-effectiveness of reconfiguration planning with different $k$ values. Plot the available capacity of

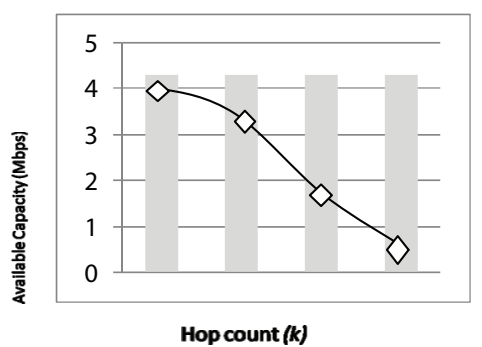

Figure 7. Impact of reconfiguration range. The hop length can help ARS search for reconfiguration plans. However, the benefit from the increased length is small, whereas the number of total changes for the reconfiguration adversely increases. 
the faulty area after reconfigurations. ALR can improve the available links' capacity by increasing the reconfiguration range. However, its improvement becomes marginal as the range increases. This saturation results mainly from the fixed number of radios of each node. In other words, the improvement is essentially bounded by the total capacity of physical radios. Furthermore, because reconfiguration plans with a larger range are required to incur more changes in network settings, the bandwidth gain per change significantly degrades (e.g., capacity gain per change at the hop count of 4 as in Figure 7.) We also observed the similar results in other reconfiguration requests $(\mathrm{T} 2, \mathrm{~T} 3, \mathrm{~T} 4)$, but omitted them for brevity.

\section{Conclusion}

This work presented an autonomous link recovery system (ALR) that enables a multiradio WMN to autonomously recover the link failures. Our implementation on ns2based simulation have demonstrated the effectiveness of ALR in recovering from local link-failures and in satisfying applications' diverse QoS demands. Furthermore, ALR effectively identifies reconfiguration plans that satisfy applications' QoS constraints, admitting up to two times more flows than static assignment, through QoS-aware planning. Next, ALR's online reconfigurability allows for real-time failure detection and network reconfiguration.

\section{References}

1. Akyildiz I, Wang W et al. (2005). Wireless mesh networks: A survey, Computer. Networks., vol 47(4), 445-487.

2. Motorola, Inc., Mesh Broadband, Schaumburg, IL, Available From: http://www.motorola.com/mesh

3. Kyasanur P, and Vaidya N H (2005). Capacity of multi-channel wireless networks: Impact of number of channels and interfaces, MobiCom '05 Proceedings of the 11th annual international conference on Mobile computing and networking, 43-57.

4. Bahl P, Chandra R et al. (2004). SSCH: Slotted seeded channel hopping for capacity improvement in IEEE 802.11 ad-hoc wireless networks, in Proceedings of ACM MobiCom, 216-230.

5. Aguayo D, Bicket J et al. (2004). Link-level measurements from an $802.11 \mathrm{~b}$ mesh network, in SIGCOMM '04 Proceedings of the 2004 conference on Applications, technologies, architectures, and protocols for computer communications, 121-132.

6. Subramanian A P, Gupta H et al. (2008). Minimum interference channel assignment in multiradio wireless mesh networks, 4th Annual IEEE Communications Society
Conference on Sensor, Mesh and Ad Hoc Communications and Networks, 2007. SECON '07, vol 7(12), 1459-1473.

7. Tanenbaum A S, and Steen M V (2002). Distributed Systems, Chapter 4, Upper Saddle River, NJ: Pearson Education, 116130 .

8. Xue Q, and Ganz A (2003). Ad hoc QoS on-demand routing (AQOR) in mobile ad hoc networks, Journal of Parallel Distributed Computing, vol 63(2), 154-165.

9. Kim K - H, and Shin K G(2009). On accurate and asymmetry-aware measurement of link quality in wireless mesh networks, Journal of IEEE/ACM Transactions on Networking (TON), vol 17(4), 1172-1185.

10. Akella A, Judd G et al. (2005). Self-management in chaotic wireless deployments, MobiCom '05 Proceedings of the 11th annual international conference on Mobile computing and networking, 185-199.

11. Kodialam M, and Nandagopal T (2005). Characterizing the capacity region in multi-radio multi-channel wireless mesh networks, MobiCom '05 Proceedings of the 11th annual international conference on Mobile computing and networking, 73-87.

12. Brzezinski A, Zussman G et al. (2006). Enabling distributed throughput maximization in wireless mesh networks: A partitioning approach, MobiCom '06 Proceedings of the 12th Annual International Conference on Mobile Computing and Networking, Los Angeles, 26-37.

13. Raniwala A, and Chiueh T (2005). Architecture and algorithms for an IEEE 802.11-based multi-channel wireless mesh network, Proceedings IEEE INFOCOM 2005, 24th Annual Joint Conference of the IEEE Computer and Communications Societies, vol 3, 2223-2234.

14. Nelakuditi S, Lee S et al. (2005). Blacklist-aided forwarding in static multihop wireless networks, 2005 Second Annual IEEE Communications Society Conference on Sensor and Ad Hoc Communications and Networks, 2005, IEEE SECON 2005, 252-262.

15. Chen S, and Nahrstedt K (1999). Distributed quality-ofservice routing in ad hoc networks, IEEE Journal on Selected Areas in Communications, vol 17(8), 1488-1505.

16. Qiu L, Bahl P et al. (2005). Troubleshooting multi-hop wireless networks, Proceedings of ACM SIGMETRICS, 380-381.

17. Kotz D, Newport C et al. (2004). Experimental evaluation of wireless simulation assumptions, Department of Computer Science, Dartmouth College, Hanover, NH, Technical Report TR2004-507.

18. Henderson T, Kotz D et al. (2004). The changing usage of amature campus-wide wireless network, Proceeding of ACM MobiCom, PA, 187-201.

19. Buddhikot M, Kolodzy $P$ et al. (2005). DIMSUMnet: New directions in wireless networking using coordinated dynamic spectrum access, Sixth IEEE International 
Symposium on a World of Wireless Mobile and Multimedia Networks, 2005, WoWMoM 2005, 78-85.

20. Braden R, Zhang L et al. (1997). Resource reservation protocol (RSVP), Internet RFC 2205 (rfc2205.txt).

21. Couto D S D, Aguayo D et al. (2003). A high-throughput path metric for multi-hop wireless routing, MobiCom '03 Proceedings of the 9th Annual International Conference on Mobile Computing and Networking, 134-146.

22. Ramanchandran K, Belding-Royer E et al. (2006). Interference- aware channel assignment in multi-radio wireless mesh networks, Proceedings INFOCOM 2006, 25th IEEE International Conference on Computer Communications, $1-12$.

23. Draves R, Padhye J et al. (). Routing in multi-radio, multi-hop wireless mesh networks, Proceedings of ACM MobiCom.

24. Perkins C, Belding-Royer E et al. (2003). Ad-hoc on-demand distance vector routing, Internet RFC 3561 (rfc3561.txt).

25. Johnson D B, and Maltz D A (1996). Dynamic source routing in ad hoc wireless networks, The Kluwer International Series in Engineering and Computer Science, vol 353, 153-181.

26. Holland G, Vaidya $\mathrm{N}$ et al. (2001). A rate-adaptiveMAC protocol for multi-hop wireless networks, MobiCom '01
Proceedings of the 7th annual international conference on Mobile computing and networking, 236-251.

27. Gross J L, and Yellen J (2009). Graph Theory and Its Applications, 2nd Edn., London, U.K, Chapman \& Hall/ CRC, 2006. Netw., vol 17, No. 4, 1172-1185.

28. Perkins C and Bhagwat P (1994). Highly dynamic destination-sequenced distance-vector routing (DSDV) for mobile computers, SIGCOMM '94 Proceedings of the conference on Communications architectures, protocols and applications, vol 24(4), 234-244.

29. Zhao J, Zheng $\mathrm{H}$ et al. (2005). Distributed coordination in dynamic spectrum allocation networks, 2005 First IEEE International Symposium on New Frontiers in Dynamic Spectrum Access Networks, 2005, DySPAN 2005, 259-268.

30. Marcus M J (2005). Real time spectrum markets and interruptible spectrum: New concepts of spectrum use enabled by cognitive radio, 2005 First IEEE International Symposium on New Frontiers in Dynamic Spectrum Access Networks, 2005, DySPAN 2005, 512-517.

31. Alicherry M, Bhatia R et al. (2005). Joint channel assignment and routing for throughput optimization in multi-radio wireless mesh networks, Proc. ACM MobiCom, Cologne, Germany, pp. 58-72. 